APAP should therefore be preferred for acute pain management.

Disclosure(s) Nothing to disclose

\section{P53 EXPOSURE OF INFANTS TO BROMINATED FLAME RETARDANTS THROUGH BREAST-MILK}

${ }^{1} \mathrm{E}$ Kohn ${ }^{*}{ }^{2} \mathrm{~S}$ Efreim, ${ }^{3,4} \mathrm{R}$ Lubetzky, ${ }^{3,4} \mathrm{D}$ Mandel, ${ }^{3,4} \mathrm{R}$ Marom, ${ }^{4,5} \mathrm{M}$ Betser, ${ }^{4,6} \mathrm{R}$ Keidar, ${ }^{4,6} \mathrm{~A}$ Livne, ${ }^{7} \mathrm{~A}$ Levy, ${ }^{8} \mathrm{P}$ Factor-Litvak, ${ }^{2} \mathrm{~S}$ Soback, ${ }^{2} \mathrm{M}$ Britzi, ${ }^{1,4} \mathrm{M}$ Berkovitch. ${ }^{1}$ Clinical Pharmacology and Toxicology, Assaf Harofeh Medical Centre, Zerifin; ${ }^{2}$ Residues Lab, Kimron Veterinary Institute, Beit-Dagan; ${ }^{3}$ Departments of Neonatology and Pediatrics, Dana Childrens' Hospital, Tel-Aviv Medical Centre; ${ }^{4}$ Sackler Faculty of Medicine, Tel-Aviv University, Tel Aviv; ${ }^{5}$ Obstetrics and Gynecology Division; ${ }^{6}$ Department of Neonatology, Assaf Harofeh Medical Centre, Zerifin; ${ }^{7}$ Department of Public Health, Ben Gurion University of the Negev, Beer Sheva, Israel; ${ }^{8}$ Department of Epidemiology, Mailman School of Public Health, Columbia University, New York, NY, USA

\subsection{6/archdischild-2019-esdppp.91}

Introduction Polybrominated Diphenyl Ethers (PBDEs) are non-biodegradable flame retardants, accumulated in biological systems and acting as endocrine disruptors. Breast feeding is a major route of exposure in infancy. Taken together with the critical development of this age and the potential adverse effects of PBDEs, it is important to monitor these contaminants in breastmilk.

Objective To evaluate the exposure of infants to PBDEs

Methods 343 families were recruited during 2013-2016 in Assaf Harofeh and Ichilov to create the AHI-EHF cohort. Maternal blood and urine, cord blood, breast milk and meconium were collected. Participants filled out questionnaires about socio-demographic status, medical history, exposures and life habits. Colostrum samples were collected from women at the maternity department. PBDEs in colostrum and Infant formulas levels were analyzed using GC-MS

Results and discussion Out of 183 serum samples, only 11 (6\%) detectable levels of PBDEs. PBDEs were found in all colostrum samples. The average concentration of total PBDEs in breastmilk was $714 \mathrm{ng} / \mathrm{L}$. PBDEs levels were also measured in three infant formulas. Unlike breastmilk, infant formulas had of only 3 congeners and levels were relatively low. The average concentration of total PBDEs in infant formulas was $153 \mathrm{ng} / \mathrm{L}$. PBDEs, were found to be negatively correlated to anno-penile index (API) which serve as a marker for endocrine disruption.

Conclusions PBDEs levels in breast milk are higher than levels in some European countries, but lower than in North America. PBDEs might have negative influence on AGD in boys. Maternal exposure to PBDEs and the significance of it should be further investigated.

Disclosure(s) Nothing to disclose

\section{P54 KIDS-STEP: A SWISS MULTI-CENTRE RCT ON EFFECTIVENESS OF ADJUNCT BETAMETHASONE THERAPY IN HOSPITALISED CHILDREN WITH COMMUNITY ACQUIRED PNEUMONIA}

\footnotetext{
${ }^{1} \mathrm{M}$ Kohns Vasconcelos*, ${ }^{1} \mathrm{R}$ Santoro, ${ }^{2} \mathrm{M}$ Coslovsky, ${ }^{1} \mathrm{~J}$ van den Anker, ${ }^{1} \mathrm{JA}$ Bielicki. ${ }^{1}$ Department for Paediatric Pharmacology, University Childrens' Hospital Basel (UKBB); ${ }^{2}$ Department of Clinical Research, Clinical Trial Unit, University and University Hospital of Basel, Basel, Switzerland
}

10.1136/archdischild-2019-esdppp.92
Background The incidence of community-acquired pneumonia (CAP) in young children is high (20-30/1000 child-years) and is associated with a high rate of hospitalisation (around 10/ 1000 child-years). In adults, a benefit of adjunct corticosteroids on time to clinical stability and hospital discharge has been observed and confirmed in systematic reviews and metaanalyses. In contrast, only few small trials have addressed the potential impact of oral steroid treatment in CAP during childhood. The purpose of this study is to concurrently evaluate whether adjunct treatment with corticosteroids in children hospitalised with CAP is more effective in terms of the proportion of children reaching clinical stability and whether such adjunct treatment is no worse in terms of CAP relapse.

Methods Children in KIDS-STEP ${ }^{1}$ receive either oral betamethasone or oral placebo dosed once daily for two consecutive days. We include 700 children from age 1 weighing at least 7 kilograms and up to a body weight of 35 kilograms and age below 10 years hospitalised for CAP using a clinical diagnosis. Co-primary outcomes are (a) The proportion of children clinically stable at 48 hours after randomisation. (b) The proportion of children with CAP-related readmission within 28 days after randomization. Secondary outcomes will be captured to further evaluate the efficacy and safety of adjunct oral steroids in the management of childhood CAP, including proportion of children experiencing solicited side effects of the trial treatment and/or serious adverse events, time to hospital discharge after index hospitalisation in days, time away from routine child care and away from work (for parents) in days up to 28 days after randomisation and total antibiotic exposure in days up to 28 days after randomisation.

Results Enrolment started in November 2018 and is currently proceeding at approximately 1 participant per participating hospital per week.

\section{REFERENCES}

1. Study registration: BASEC - EKNZ 2018-00563

Disclosure(s) Nothing to disclose

\section{P55 PREVENTING INAPPROPRIATE HYDROXYUREA DOSING IN CHILDREN BY INTRODUCING A CHILD-APPROPRIATE PREPARATION}

G Koren*. Maccabi Research Institute, Tel Aviv, Israel

\subsection{6/archdischild-2019-esdppp.93}

Background Hydroxyurea (HU) is the only FDA- approved disease- modifying drug for sickle cell disease, by inducing the production of fetal hemoglobin and thus decreasing the sickling of red blood cells. Till recently HU was available only in adult doses of $1000 \mathrm{mg}$. This meant that to aim at the standard dose of $20 \mathrm{mg} / \mathrm{kg} / \mathrm{d}$, most young children had to be overdosed, or the dose had to be fluctuated daily to achieve the aimed mean dose. Because adherence improves with unchanged daily dose, and due to the more than 10 fold variability in HU pharmacokinetics in children, there was an urgent need for a pediatric formulation of HU.

Methods and results This issue has been solved with the FDA approval of the French-originated orphan HU, Siklos, a preparation of 50 and $100 \mathrm{mg}$, which prevents the risk of inappropriate dosing in children.

Studies show that the child appropriate dose preparation much more closely allow young children to receive 